\title{
Chapter 11 \\ Modeling the Role of Microplastics \\ in Bioaccumulation of Organic Chemicals \\ to Marine Aquatic Organisms. A Critical \\ Review
}

\author{
Albert A. Koelmans
}

\begin{abstract}
It has been shown that ingestion of microplastics may increase bioaccumulation of organic chemicals by aquatic organisms. This paper critically reviews the literature on the effects of plastic ingestion on the bioaccumulation of organic chemicals, emphasizing quantitative approaches and mechanistic models. It appears that the role of microplastics can be understood from chemical partitioning to microplastics and subsequent bioaccumulation by biota, with microplastic as a component of the organisms' diet. Microplastic ingestion may either clean or contaminate the organism, depending on the chemical fugacity gradient between ingested plastic and organism tissue. To date, most laboratory studies used clean test organisms exposed to contaminated microplastic, thus favouring chemical transfer to the organism. Observed effects on bioaccumulation were either insignificant or less than a factor of two to three. In the field, where contaminants are present already, gradients can be expected to be smaller or even opposite, leading to cleaning by plastic. Furthermore, the directions of the gradients may be opposite for the different chemicals present in the chemical mixtures in microplastics and in the environment. This implies a continuous trade-off between slightly increased contamination and cleaning upon ingestion of microplastic, a trade-off that probably attenuates the overall hazard of microplastic ingestion. Simulation models have shown to be helpful in mechanistically analysing these observations and scenarios, and are discussed in detail. Still, the literature on parameterising such models is limited and further experimental work is required to better constrain the parameters in these models for the wide range of organisms and chemicals acting in the aquatic environment. Gaps in knowledge and recommendations for further research are provided.
\end{abstract}

\footnotetext{
A.A. Koelmans $(\triangle)$

Aquatic Ecology and Water Quality Management Group, Department of Environmental Sciences, Wageningen University, P.O. Box 47, 6700 AA Wageningen, The Netherlands e-mail: bart.koelmans@wur.nl
}

\begin{abstract}
A.A. Koelmans
IMARES-Institute for Marine Resources and Ecosystem Studies, Wageningen UR,

P.O. Box 68, 1970 AB IJmuiden, The Netherlands
\end{abstract}


Keywords Additives - Bioaccumulation - Chemical transfer - Microplastic - Persistent organic pollutants

\subsection{Introduction}

Pollution with plastic debris and microplastic fragments has been recognized as a major problem in fresh water and marine systems (Derraik 2002; Andrady 2011; Koelmans et al. 2014a). Negative effects may relate to entanglement in plastic wires or nets, or to ingestion, which has been reported for benthic invertebrates, birds, fish, mammals and turtles. Extensive overviews of the deleterious effects of litter on marine life are provided by Kühn et al. (2015) and by Lusher (2015). Furthermore, it is generally assumed that microplastic may act as a vector for transport of chemicals associated with the plastic particles, such as persistent organic pollutants (POPs) or additives, residual monomers or oligomers of the component molecules of the plastics (hereafter referred to as 'additives') (Gouin et al. 2011; Teuten et al. 2007, 2009; Hammer et al. 2012; Browne et al. 2013; Rochman 2015; Lusher 2015). Hydrophobic chemicals including polychlorobiphenyls (PCB), polycyclic aromatic hydrocarbons (PAH) or polybrominated diethyl ethers (PBDEs), are known to concentrate in polymers such as polyvinylchloride (PVC), polyethylene (PE), polystyrene (PS) or polyoxymethylene (POM), which is the basis of using the latter materials in passive sampling devices (e.g. Hale et al. 2010). Microplastic particles present in seas and oceans have been found to contain considerable quantities of these chemicals (e.g. Ogata et al. 2009; Hirai et al. 2011). Concentrations of additives such as nonylphenol (NP), bisphenol A (BPA), PBDEs and phthalates also have been reported to be high in marine plastics, rendering them a potential source to the environment and marine biota. The question whether microplastic-mediated chemical transfer poses a serious actual hazard, however, depends on several other factors. First, for transport of the chemicals from plastic to an organism, a gradient that drives the chemical from plastic to the organism is required (Gouin et al. 2011; Koelmans et al. 2013a, b). If, however, a reverse gradient existed, ingestion would lead to cleaning of the organism and ingestion would in this sense be beneficial. Second, the chemical uptake through ingestion of plastic should be substantial compared to other exposure pathways, i.e. by food ingestion or uptake from ambient water. Because POPs as well as additives are ubiquitous in many environments, a dominant role of plastic ingestion is not self-evident (Koelmans et al. 2014b). Third, the chemical hazard of microplastic ingestion should relate to all the chemicals in the plastic-organism system, that is, the chemical mixture transferred to or from the organism by ingestion and chemicals should not be considered in isolation. A plastic additive may leach from a heavily contaminated plastic particle, but clean the organism from its body burden of legacy POPs at the same time. This means that there may be a trade-off between positive and negative effects of microplastic ingestion. 
To date, a few controlled experimental studies have been published confirming transfer of chemicals from microplastic to marine organisms. Besseling et al. (2013) mimicked natural conditions by exposing relatively clean worms to mixtures of a natural marine sediment and PS microplastic, which were preequilibrated with PCBs, thus providing realistic exposure conditions. The presence of microplastic caused a small (factor of three) increase in bioaccumulation. However, bioaccumulation decreased again at higher concentrations. The authors argued that PS may not have caused PCB transfer but that the increased bioaccumulation probably had a biological cause, such as a change in lipid content or feeding rates. Browne et al. (2013) did not use natural sediment but exposed clean lug worms (Arenicola marina) to sand with $5 \%$ of PVC microplastic that was presorbed with high concentrations of nonylphenol, phenanthrene, triclosan and/or PBDE-47. Because by using clean worms, a gradient from the PVC to the organism was created, chemical transfer from the particles to the worms occurred, but uptake from sand was larger than that from the PVC microplastic. Rochman et al. (2013b) exposed fish (Japanese medaka; Oryzias latipes) to contaminated food, to contaminated food mixed with $10 \%$ virgin low density PE (LDPE) and to contaminated food mixed with LDPE that was pre-equilibrated in seawater. They observed an increase in body burdens up to a factor of 2.4 after two months, which was statistically significant for chrysene, PCB28 and most PBDEs. Chua et al. (2014) observed that adding PBDE-spiked microplastics to seawater with amphipods (Allorchestes compressa) in closed vials resulted in PBDE uptake by the amphipods, which was however only statistically significant compared to the controls when spiked concentrations were ten times higher than environmentally relevant concentrations. Addition of clean plastic to the same closed systems yet pre-contaminated with PBDEs resulted in a decreased uptake.

Considering the complex processes involved, modelling approaches have been proven useful for the interpretation of experimental data as well as for prognostic assessments of the possible hazards caused by plastic ingestion. Model-based scenario studies have helped to define in which cases plastic ingestion may be relevant, dependent on plastic type, chemical properties and species traits. The aim of this chapter is to present and critically discuss the model approaches used to quantify the effect of plastic on bioaccumulation of POPs and additives. This includes a mathematical description of the processes at play, a review of the model-based inferences described in the literature, and an outlook to future work and recommendations.

\subsection{Models to Assess the Importance of Microplastic Ingestion}

In the literature several processes have been identified as important to address when modelling effects of microplastic on the bioaccumulation of chemicals. These studies typically consider biota lipids as the target tissue for chemical 
accumulation. First of all, plastic has been reported to act as an additional sorbent for POPs and additives (Andrady 2011). Upon addition of clean plastic in any closed system, chemicals will bind to the plastic thus lowering the chemical concentration in other media or compartments present, such as water, sediment and biota (Teuten et al. 2007, 2009; Gouin et al. 2011; Koelmans et al. 2013a; Chua et al. 2014). This mechanism of repartitioning thus causes a decrease in exposure of aquatic organisms to chemicals in water, sediment organic matter and food. Conversely, if the plastic carries high enough concentrations of chemicals to act as a source, these chemicals will be released and redistribute among the various media present, possibly increasing the chemical concentrations in the other compartments, including biota (Hammer et al. 2012; Koelmans et al. 2014b). Consequently, whether plastic acts as a source or a sink depends on the gradient between the chemical concentration in the plastic and the ambient water. Second, plastic items may slowly disintegrate and degrade under the influence of turbulence and UV radiation or by microbial activity (Andrady 2011, 2015). This means that the chemical mass held by the plastic being degraded will be released, even if no a priori gradient between the chemical concentration in plastic and in ambient water exists. In turn, smaller and/or weathered plastic items may have different sorption properties compared to their pristine original state (Teuten et al. 2009; Rochman et al. 2013a). Leaching and weathering for instance may change the polymers' structure and overall polarity. For smaller particles, surface sorption may become dominant over bulk partitioning, a phenomenon that probably is most relevant for polymer particles reaching the nano-scale (Velzeboer et al. 2014; Koelmans et al. 2015). Third, ingestion will bring plastic particles inside the gastrointestinal tract (GIT) of marine organisms where they will stay for a period of time depending on the biology of the species. Whether chemicals are being transferred inside the GIT primarily depends on the gradient between chemical fugacity in the plastic and in the relevant organisms' tissue, which for POPs and hydrophobic chemicals in general, especially is the lipids. If there is no gradient, plastic will pass the GIT and leave the organism without any chemical transfer. The possibility that there is a positive gradient between plastic and lipids receives a lot of speculation in the literature because it would imply an increase of exposure to plastic-associated chemicals compared to a scenario without ingestion of microplastic (Teuten et al. 2007, 2009; Hammer et al. 2012; Chua et al. 2014; Rochman 2015). However, the reverse, i.e. a gradient towards plastic, may be evenly likely (Gouin et al. 2011; Koelmans et al. 2013a; Chua et al. 2014). This potential uptake pathway assumes that microplastics are not decomposed in the relative short GIT residence time of hours to days for most species. Only for really large items that cause obstruction and blockage of the GIT as is observed for instance for birds, decomposition may become relevant. In such a scenario, however, physical harm would probably cause stress and mortality earlier than that related to chemical release. Consequently, an essential difference in chemical risk originating from contaminated plastic versus that of contaminated food as a diet component is that pre-equilibrated food is digested, which leads to an immediately increased concentration (fugacity) inside the GIT, whereas pre-equilibrated plastic may leave the 
GIT unchanged in many cases. If pre-equilibrated food and plastic are ingested as a mixture, the pulse exposure due to decomposition of the food inside the GIT will cause a gradient from the gut and biota lipids towards the plastic. This means that plastic ingestion can suppress biomagnification and that plastic ingestion in fact may clean the organism (Gouin et al. 2011; Koelmans et al. 2013a). Because regular biomagnification increases with trophic level, the gradient between biota lipids and plastic would be larger for higher trophic levels, leading to more transfer from biota lipids to the plastic. Finally, regardless of whether microplastic increases or attenuates bioaccumulation, the actual importance of plastic ingestion also depends on whether the percentage of chemical transfer due to microplastic ingestion is substantial compared to that of the other uptake pathways, such as the transfer from digested food and uptake from water. This importance in turn depends on the residence times and ingestion rates of plastic and food items in the GIT and the exchange kinetics between these items and gut fluids. In summary, the effects of microplastic on bioaccumulation can be understood from (a) changes in external exposure driven by competitive partitioning processes and (b) by changes in 'internal' exposure due to microplastic acting as a source or a sink depending on initial concentrations in plastic and biota lipids that determine the direction of the gradient. Several authors have provided mathematical process descriptions and parameters to quantify the processes mentioned and to unify them in an integrated model framework. Below, the most important approaches are provided and reviewed.

\subsubsection{Equilibrium Partitioning}

Addition of clean microplastic to a closed contaminated system will cause a gradient towards the plastic and thus lower the concentrations in the compartments present, for instance water and biota, until a new equilibrium is established. Adding contaminated plastic will cause the opposite process and lead to higher concentrations in water and biota. The kinetics of such a systems response is well-understood and depends on the response times for the individual exchange processes, i.e. water-sediment, water-biota and water-plastic. In general, the slowest process will be rate-determining. For polymers in water, kinetics depends on the resistances to transfer, which are the resistance due to polymer diffusion and the resistance due to the undisturbed boundary layer (UBL) surrounding plastic particles. For hydrophobic chemicals and plastic particles $>1 \mathrm{~mm}$, it is generally assumed that the UBL resistance dominates and transfer can be described by (Schwarzenbach et al. 2003):

$$
\frac{d C_{P L}}{d t}=k_{1} C_{W}-k_{2} C_{P L}
$$

where $\mathrm{t}=$ time and $\mathrm{k}_{1}\left(\mathrm{~L} \mathrm{~kg}^{-1} \mathrm{~d}^{-1}\right)$ and $\mathrm{k}_{2}\left(\mathrm{~d}^{-1}\right)$ are first-order rate constants that can be related to the thickness of the UBL and aqueous diffusivity of the chemical, 
and $C_{P L}(\mu \mathrm{g} / \mathrm{kg})$ and $C_{W}(\mu \mathrm{g} / \mathrm{L})$ are concentrations in plastic and water, respectively. The presence of biofilms on the plastic may slow down the exchange kinetics. In systems with excess of water and sediment, $C_{W}$ will not decrease due to sorption to plastic and can be assumed constant, i.e. $C_{W, 0}$ such that:

$$
C_{P L}=C_{W, 0} \frac{k_{1}}{k_{2}}\left(1-e^{-k_{2} t}\right)
$$

where $\mathrm{k}_{1} / \mathrm{k}_{2}$ is the plastic to water partition coefficient $\mathrm{K}_{\mathrm{P}, \mathrm{PL}}(\mathrm{L} / \mathrm{kg})$, which may differ for different types of plastics. For chemicals that are less hydrophobic, exchange may be driven by polymer diffusion, a process that follows Fick's $2^{\text {nd }}$ law of diffusion, which for spherical particles reads (Schwarzenbach et al. 2003; Teuten et al. 2009; Endo et al. 2013):

$$
\frac{d C_{P L}}{d t}=\frac{D_{e f f}}{r^{2}} \frac{\delta}{\delta r}\left(r^{2} \frac{\delta C_{P L}}{\delta t}\right)
$$

Here, the key parameters are $D_{\text {eff }}\left(\mathrm{m}^{2} \mathrm{~d}^{-1}\right)$, the effective polymer diffusion coefficient and $\mathrm{r}(\mathrm{m})$, the radius of the plastic particle. Although fundamentally different, the modelling of the two regimes can be unified using the approximation (Schwarzenbach et al. 2003; Koelmans et al. 2013a, b):

$$
k_{2} \cong 23 D_{e f f} / r^{2}
$$

In practice, half-lives (i.e. $\mathrm{t}_{1 / 2}=0.693 / \mathrm{k}_{2}$ ) for desorption from microplastic particles in seawater have been reported as one day to years (Teuten et al. 2007; Endo et al. 2013; Bakir et al. 2014; Rochman et al. 2013a) depending on the chemical, the plastic, stirring conditions, presence of dissolved organic matter and measurement method. Because of the environmental persistence of microplastics, most particles will have resided in the water for years or decades and thus can generally be assumed to be close to sorption equilibrium.

The effect of addition of plastic on the aqueous chemical concentration in a simple closed sediment-water system can be calculated from a mass balance, thus: $C_{W}^{1}\left(V_{W}+M_{S E D} K_{P, S E D}\right)=C_{W}^{2}\left(V_{W}+M_{S E D} K_{P, S E D}+M_{P L} K_{P, P L}\right)$, which translates into:

$$
\frac{C_{W}^{2}}{C_{W}^{1}}=\frac{V_{W}+M_{S E D} K_{P, S E D}}{V_{W}+M_{S E D} K_{P, S E D}+M_{P L} K_{P, P L}}
$$

in which $C_{W}^{1}$ and $C_{W}^{2}$ are the chemical concentrations in water before and after the addition of microplastic ( $\mu \mathrm{g} / \mathrm{L}), M_{S E D}$ and $M_{P L}$ are masses of sediment and plastic $(\mathrm{kg})$, and $K_{P, S E D}$ is the sediment-water equilibrium partition coefficient. For the sake of simplicity, only sediment is considered here, but for hydrophobic chemicals, similar terms $M_{i} K_{P, i}$ should be added for other important compartments ' $\mathrm{i}$ ' such as phytoplankton and dissolved organic matter (DOC). Gouin et al. (2011) provided the most elaborate analysis in this respect by also including the air compartment using air-volume and air-water partition coefficients. It follows from 
Eq. (11.5) that addition of plastic will only be important if the term $M_{P L} K_{P, P L}$ in the denominator of (11.5) adds substantially to the terms $V_{W}+M_{S E D} K_{P, S E D}$ and similar terms $M_{i} K_{P, i}$ for phytoplankton and DOC.

\subsubsection{Decomposition and Disintegration}

Decomposition, disintegration or (bio)degradation have been reported to occur at time scales of years to decades (Andrady 2011). Recent laboratory studies report degradation of $1-1.75 \%$ of low density PE mass in $30 \mathrm{~d}$, for micro-organisms isolated from marine waters and with high microbial densities (Harshvardhan and Jha 2013). If surface oxidation or surface degradation is the rate-limiting step, overall degradation can be assumed to depend on the amount of surface area that is available. With ongoing degradation, the surface area per unit of volume will increase due to increased surface roughness, as well as reduced particle size. The shrinking-particle theory (e.g. Di Toro et al. 1996) accounts for this change in size and for mono-disperse spherical particles would predict:

$$
V_{t}=V_{0}\left(1-\frac{2 k_{s} t}{d_{0}}\right)^{\alpha}
$$

in which $V_{t}\left(\mathrm{~m}^{3}\right)$ is the particle volume at time $\mathrm{t}, V_{0}\left(\mathrm{~m}^{3}\right)$ is the initial particle volume, $d_{0}(\mathrm{~m})$ is the initial particle diameter (spheres) or thickness (polymer films), $\alpha$ is a particle shape factor ( $\alpha=3$ for spheres and $\alpha=1$ for thin films) and $\mathrm{k}_{\mathrm{s}}$ is the apparent shrinking-rate constant $\left(\mathrm{m}^{3} \mathrm{~m}^{-2} \mathrm{~d}^{-1}\right)$. Calibration of the model on the $\sim 1 \%$ PE mass loss in $30 \mathrm{~d}$ observed for thin films deployed by Harshvardhan and Jha (2013) (with $\alpha=1$ and assuming an initial thickness of $25.4 \mu \mathrm{m}$ (1 mil) for their PE film), would yield a low value for $\mathrm{k}_{\mathrm{s}}$ of $4.2 \times 10^{-9} \mathrm{~m}^{3} \mathrm{~m}^{-2} \mathrm{~d}^{-1}$. It can be assumed that loss of polymer equates to loss of chemical held by that volume of polymer. The time scales at which these decomposition processes occur, however, probably are orders of magnitude longer than the time scales of plastic-water partitioning or transfer inside the organisms' gut (see below). This implies that decomposition is not directly relevant for bioaccumulation assessment.

\subsubsection{Bioaccumulation}

Bioaccumulation can be modelled using traditional approaches that use a mass balance of uptake and loss processes (e.g. Thomann et al. 1992; Hendriks et al. 2001) (Fig. 11.1). Extensions of these models to account for uptake from contaminated particles as diet components were first provided by Sun et al. (2009) and Janssen et al. (2010). Koelmans et al. (2013a, b, 2014b) modelled bioaccumulation of hydrophobic chemicals $\left(\mathrm{dC}_{\mathrm{B}, \mathrm{t}} / \mathrm{dt}\right.$; $\left.\mu \mathrm{g} \times \mathrm{kg}^{-1} \mathrm{~d}^{-1}\right)$ from an environment containing plastic using: 


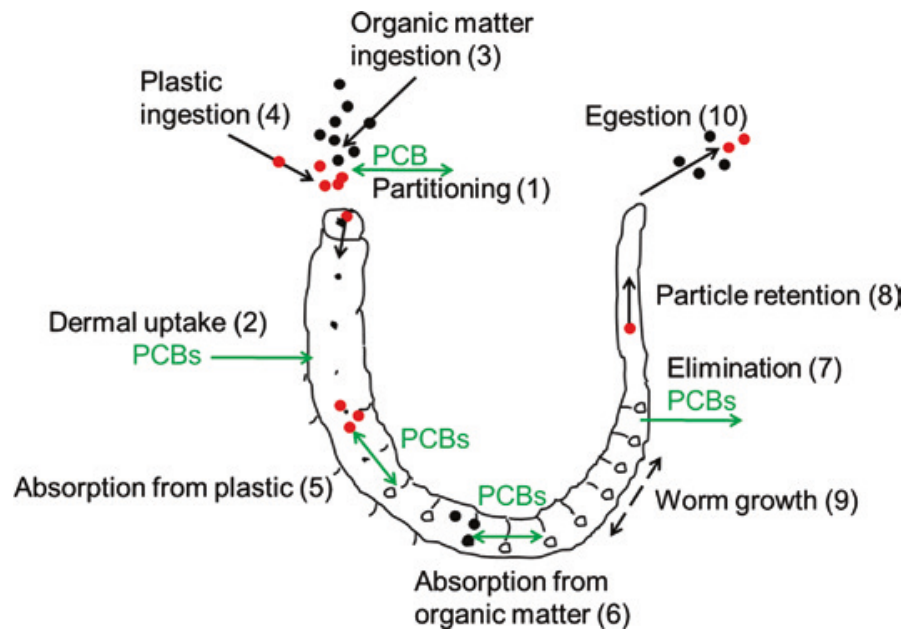

Fig. 11.1 Schematic representation of processes required for plastic-inclusive bioaccumulation modeling (example for PCBs accumulation in a lugworm Arenicola marina): 1 Partitioning between plastic, sediment and water, 2 dermal uptake, 3 organic matter (food, biofilm) ingestion, 4 microplastic ingestion, 5 absorption from plastic, 6 absorption from organic matter, 7 elimination, 8 particle retention, 9 worm growth, 10 particle egestion (sediment and plastic). Same or similar process descriptions can be used for other marine/aquatic organisms. Reprinted with permission from Koelmans et al. (2013a). Copyright 2013 American Chemical Society

$$
\frac{\mathrm{dC}_{\mathrm{B}, \mathrm{t}}}{\mathrm{dt}}=\mathrm{k}_{\mathrm{derm}} \mathrm{C}_{\mathrm{W}}+\mathrm{IR}\left(\mathrm{S}_{\mathrm{FOOD}} \mathrm{a}_{\mathrm{FOOD}} \mathrm{C}_{\mathrm{FOOD}}+\mathrm{S}_{\mathrm{PL}} \mathrm{C}_{\mathrm{PLR}, \mathrm{t}}\right)-\mathrm{k}_{\text {loss }} \mathrm{C}_{\mathrm{B}, \mathrm{t}}
$$

where the first term quantifies dermal (for fish; including gills) uptake from water. The second term quantifies uptake from the diet and exchange with plastic particles. The third term quantifies overall loss due to elimination and egestion. The first and third term can be parameterised following traditional approaches with $\mathrm{k}_{\mathrm{derm}}\left(\mathrm{L} \times \mathrm{kg} \times \mathrm{d}^{-1}\right)$ and $\mathrm{k}_{\text {loss }}\left(\mathrm{d}^{-1}\right)$, first-order rate constants for dermal uptake and overall loss through elimination and egestion. In the second term, IR $\left(\mathrm{g} \times \mathrm{g}^{-1} \times \mathrm{d}^{-1}\right)$ represents the mass of food ingested per unit of time and organism dry weight, aFOOD is the absorption efficiency from the diet, $\mathrm{S}_{\mathrm{FOOD}}$ and $S_{P L}$ are the mass fractions of food and plastic in ingested material, respectively $\left(\mathrm{S}_{\mathrm{FOOD}}+\mathrm{S}_{\mathrm{PL}}=1\right)$ and $\mathrm{C}_{\mathrm{FOOD}}$ is the chemical concentration in the diet. The product $\mathrm{a}_{\mathrm{FOOD}} \times \mathrm{C}_{\mathrm{FOOD}}$ quantifies the contaminant concentration that is transferred from food, i.e. prey, to the organism during gut passage. The plastic particles may contain a biofilm (BF), which may also carry chemicals. The biofilm would contribute to the pool of digestible organic matter and may therefore be covered either by the sediment term or by an optional additional term in Eq. 11.7, similar to the sediment ingestion term (e.g. $I R \times S_{B F} \times a_{B F} \times C_{B F}$ ). Where regular bioaccumulation models assume digestion of diet components and thus assume a certain fixed chemical absorption efficiency, Koelmans et al. (2013a, b, 2014b) assumed plastic 
not to degrade in the short time scale of gut passage. The transferred concentration from plastic during gut passage ( $\mathrm{C}_{\mathrm{PLR}, \mathrm{t}}, \mu \mathrm{g} / \mathrm{kg}$ ) thus was modelled to be dependent on the concentrations in plastic and biota lipids, the kinetics of transfer between plastic and lipids and the GIT residence time (GRT) (see Koelmans et al. 2013a, b for detailed derivation):

$$
C_{P L R, t}=\frac{k_{1 G} C_{P L}-k_{2 G} C_{L, t}}{k_{1 G}+\frac{M_{P L}}{M_{L}} k_{2 G}}\left(1-e^{-\left(k_{1 G}+\frac{M_{P L}}{M_{L}} k_{2 G}\right) G R T}\right)
$$

in which $\mathrm{k}_{1 \mathrm{G}}$ and $\mathrm{k}_{2 \mathrm{G}}\left(\mathrm{d}^{-1}\right)$ are forward and backward first-order rate constants describing the transport between plastic and biota lipids inside the GIT. If the numerator term $k_{1 G} C_{P L}-k_{2 G} C_{L, t}$ in Eq. 11.8 is positive, transfer from the plastic to biota lipids occurs, whereas opposite transfer ('cleaning by plastic') occurs when the term is negative. Various authors have provided these $\mathrm{k}$ values at simulated gut conditions, showing about an order of magnitude enhancement of transfer rates in artificial gut fluids up to $\mathrm{k}_{1 \mathrm{G}}=10-12 \mathrm{~d}^{-1}$ (Teuten et al. 2007; Bakir et al. 2014). GRT is gut residence time (d), $\mathrm{C}_{\mathrm{PL}}$ and $\mathrm{C}_{\mathrm{L}, \mathrm{t}}(\mu \mathrm{gg} / \mathrm{kg})$ are the chemical concentrations in the ingested plastic particle and the biota lipids at the moment of ingestion and $\mathrm{M}_{\mathrm{PL}}$ and $\mathrm{M}_{\mathrm{L}}$ are the mass of plastic and lipids in the organism, respectively $(\mathrm{kg})$. If $\mathrm{C}_{\mathrm{W}}$ is constant in time (Eqs. 11.2 and 11.5) and $\mathrm{C}_{\mathrm{PL}}$ is estimated by Eq. 11.2, an analytical solution to Eqs. 11.7 and 11.8 is available that calculates the body burden at steady state $\left(\mathrm{C}_{\mathrm{B}}^{\mathrm{SS}}\right)$ (Koelmans et al. 2014b):

$$
C_{B}^{S S}=\frac{k_{\text {derm }} C_{W, 0}+I R\left(S_{F O O D} a_{F O O D} C_{F O O D}+S_{P L} k_{1 G} C_{P L} A_{P L}\right)}{I R S_{P L} k_{2 G} A_{P L} / f_{\text {lip }}+k_{\text {loss }}} \text { and } A_{P L}=\frac{1-e^{-\left(k_{1 G}+\frac{M_{P L}}{M_{L}} k_{2 G}\right) G R T_{t}}}{k_{1 G}+\frac{M_{P L}}{M_{L}} k_{2 G}}
$$

Note, that Eq. 11.9 accounts for all uptake and loss pathways and can be used to assess the relative importance of plastic ingestion as an uptake pathway compared to other pathways such as food ingestion and dermal uptake, as well as the importance of chemical loss by plastic egestion compared to regular loss mechanisms.

\subsection{Model-Based Assessment of Implications and Risks of Plastic-Associated Chemicals}

Various authors used the aforementioned concepts to assess the effects and importance of plastic-associated chemicals on chemical partitioning and bioaccumulation. This section reviews these studies. Teuten et al. (2007) modelled the effect of adding 'clean' plastic to a sediment-water system $(1.5 \mathrm{~kg}$ sediment, $0.4 \mathrm{~L}$ water, $1.5 \mathrm{~g}$ lugworm A. marina) contaminated with phenanthrene as a model compound using an equilibrium partitioning approach (Eq. 11.5). They concluded 
that plastic addition would reduce bioavailability due to scavenging of phenanthrene by the plastic. The effect was small (13\%) and depended on factors such as partition coefficients of the plastic and sediment, which also follows from Eq. 11.5. In another scenario, they assumed chemical concentrations in microplastic to be much higher in the sea-surface micro layer (SML) than in the water column. Subsequent settling and exposure of sediment biota to these enriched microplastic particles would lead to increased bioaccumulation. Both scenarios, however, used equilibrium partitioning concepts only and did not yet consider sediment and/or plastic ingestion as a possible uptake pathway. This implies that attenuation of bioaccumulation was not accounted for. There may also be some uncertainty related to the acclaimed enrichment in the SML. Analysis of the SML by Hardy et al. $(1988,1990)$ did not use passive samplers that would have detected the truly dissolved concentrations, but used analysis of total concentrations after filtration. Filtration is known to be insufficient in removing DOC and colloids present (e.g. Gschwend and Wu 1985). Because the SML is enriched with DOC, organic colloids, micro-organisms or oil films that act as 'extracting agents', this explains the enhanced apparent concentrations in the SML (e.g. Wurl et al. 2006). This is also consistent with SML concentration enrichment factors usually being higher for coastal areas and bays that have higher DOC levels, and for more hydrophobic chemicals. The truly dissolved chemical concentrations in the SML, however, would still be equal or close to those in the bulk of the water column, thus preventing enrichment of concentrations in microplastic. Furthermore, if an enhancement of concentrations in microplastics compared to the water column would still occur, desorption would probably attenuate the gradient upon settling in the water column and burial in the sediment.

Gouin et al. (2011) also used equilibrium partitioning concepts to define the chemical distribution of POPs among air, water, sediment and plastic, and used steady-state bioaccumulation modelling to assess their subsequent fate in the food web. Instead of considering one chemical their analysis spanned a wide range of chemical hydrophobicities and air-water partition coefficients. A model environment was defined representative of a coastal marine ecosystem with a realistic input of plastic debris. Mass-balance equations were used to construct chemical space diagrams. Data analysis showed that partitioning to PE was negligible $(<0.1 \%$ of chemical mass). Only if it was assumed that the present estimate of PE abundance was enhanced by three orders of magnitude and that the water contained no organic matter (i.e. DOC or phytoplankton) PE would became important ( $>1 \%$ sorption to PE) for POPs with $\operatorname{LogK}_{\mathrm{ow}}>5$. This implies that present plastic loadings were calculated to be insufficient to cause a meaningful redistribution of POPs from the oceanic environment to the plastic. Furthermore, DOC and phytoplankton that compete with plastic for POP distribution should be accounted for in order to assess whether future accumulation of plastic could lead to a substantial redistribution of POPs. Gouin et al. (2011) also discussed effects of PE presence on bioaccumulation by piscivorous fish, by including contaminated $\mathrm{PE}$ as a diet component in an elaborate food web bioaccumulation model. A steady-state approach was used that did not yet consider the kinetics of 
desorption from the plastic inside the gut, in relation to gut retention time. This means that the direction of an effect of PE ingestion would be calculated correctly but that its magnitude may have been overestimated because the model could not account for the extent of non-equilibrium in the gut. Interestingly, the authors found a counterintuitive decrease in predicted body burden upon an increase in $\mathrm{PE}$ in the diet. This was explained by the fact that without plastic, food organic matter is digested leading to high concentrations in the gut that subsequently are transferred to the organisms' lipids. In the presence of plastic, however, which is not degraded, a gradient from lipids towards plastic exists, leading to cleaning of the organism by the plastic.

Koelmans et al. (2013a, b) presented a general POP bioaccumulation model framework for marine aquatic organisms combining Eqs. 11.1-11.9, which was implemented for A. marina (Fig. 11.1). The model accounted for dilution of exposure concentration by sorption of POPs to plastic (POP 'dilution'), increased bioaccumulation by ingestion of plastic containing POPs ('carrier'), and decreased bioaccumulation by ingestion of clean plastic ('cleaning'). Kinetics in the gut were explicitly taken into account. The model was evaluated against bioaccumulation data from laboratory bioassays with PS microplastic. Further scenarios included PE microplastic, nano-sized plastic and open marine systems. Scenario studies assumed equilibrium of organisms and plastics prior to ingestion, as would occur for POPs in the environment. Model analysis showed that PS will have a decreasing effect on bioaccumulation, governed by dilution. For stronger sorbents such as polyethylene, the dilution, carrier and cleaning mechanism were more substantial. In closed laboratory bioassay systems, dilution and cleaning dominated, leading to decreased bioaccumulation. Also, in open marine systems a decrease was predicted due to a cleaning mechanism that counteracts biomagnification, similar to that recognized earlier by Gouin et al. (2011). However, the differences were considered too small to be relevant from a risk assessment perspective.

Pollution by POPs is diffuse, which implies that POPs will be always present at background concentrations, often at solid phase-water equilibrium (Van Noort and Koelmans 2012). In the early life stages of organisms, POP concentrations in the organism will be in equilibrium with the ambient water too, which implies that ingestion of polluted microplastic will coincide with the ingestion of polluted food, rendering the contribution of microplastic relatively unimportant. For additives, however, plastic ingestion by marine organisms may potentially be more relevant than for diffusely spread POPs because the plastic could still be a source of the additives (Teuten et al. 2009; Hammer et al. 2012; Koelmans et al. 2014b). Furthermore, compared to worms, leaching of additives or residual monomers may be more relevant for larger and longer-lived species, with longer gut retention times, such as fish. Two recent controlled laboratory studies confirmed that dietary exposure of organisms to microplastic pre-adsorbed with POPs or additives leads to chemical transfer from the microplastic to the organism (Browne et al. 2013; Rochman et al. 2013b). A remaining question, however, is what the relative importance of this microplastic uptake pathway is under natural conditions, where other pathways like dermal uptake, uptake via the gills or consumption of natural 
prey play a role. Furthermore, it is plausible that in actual marine systems, background chemical concentrations in biota may already exceed the concentrations that microplastic ingestion would be able to explain, in which case no gradient for transfer would exist.

In a follow up study, Koelmans et al. (2014b) used the same biodynamic model as was used for POPs to assess the potential of leaching of nonylphenol (NP) and bisphenol A (BPA) in the intestinal tracts of lugworm (A. marina) and cod (Gadus morhua). Parameters for the lugworm were based on Besseling et al. (2013). Parameters for cod were based on actual abundances of microplastic particles in the cod GIT as observed by Foekema et al. (2013). The resulting model was validated against the data provided by Browne et al. (2013) for leaching of NP from PVC to A. marina. Then, the model was used to calculate the body burdens that could be explained from plastic ingestion, which were compared to NP and BPA body burdens actually measured in the field. Uncertainty in the most crucial parameters was accounted for by probabilistic modelling. The conservative analysis showed that plastic ingestion by the lugworm indeed results in chemical transfer to the organism, but yields NP and BPA concentrations that stay below the lower ends of global NP and BPA concentration ranges in the lugworm, and therefore is unlikely to constitute a relevant exposure pathway. A similar comparison showed that plastic ingestion is also likely to constitute a negligible exposure pathway for cod.

Note that the key model concepts of chemical transfer in the intestinal tract or segments of the intestinal tract as condensed in Eqs. 11.7-11.9 are also applicable to higher marine organisms. They would only need different parameterizations and different initial boundary conditions.

\subsection{Summarizing Discussion and Recommendations}

This chapter discussed the present state of the art in modelling chemical transfer between microplastic and biota in relation to the experimental data available. Whereas the experimental data and field observations serve as best available proof of the actual occurrence of transfer processes that have been speculated on in the literature for a long time, model analysis has helped to understand why these effects occur, and to quantify their magnitude and direction. General prognostic risk assessments regarding plastic-associated chemicals will need simulation models for the same reasons why models are needed in general PBT assessment (Weisbrod et al. 2009).

Generally, the present experimental studies and model studies are consistent in that they can predict up to a factor of two to three increase in bioaccumulation if microplastic is the only source of the chemical and the only pathway of uptake. Conversely, they predict a decrease in bioaccumulation when chemical dilution outcompetes transfer in the gut. If more environmentally relevant scenarios are 
considered, i.e. with pre-equilibrated systems and all exposure pathways are accounted for, ingestion of microplastics seems to be much less important than the existing pathways. This does not mean that the hazards of plastic-associated chemicals are less than anticipated, but it may imply that the relevance of plastic ingestion as an additional exposure pathway may be less relevant than what has been assumed in the literature (e.g. Teuten et al. 2007, 2009; Hammer et al. 2012; Browne et al. 2013; Chua et al. 2014), at least for POPs.

Chemical transfer effects should not be studied or interpreted from chemical principles alone assuming biota to be a constant factor. Plastic ingestion may cause physical stress, for instance due to blockage of the GIT or decreased overall food quality (Lusher 2015), which in turn may affect ingestion rates, lipid contents, growth rates and in turn kinetic parameters for chemical transfer. Distinguishing between these chemical and biological effect mechanisms is an important challenge when interpreting bioaccumulation data from the laboratory or the field.

Although considerable progress has been made over the past years, there still is only a hand full of bioaccumulation studies addressing transfer from microplastic, typically of a 'proof of principle' nature. The processes at play seem to be well understood, their parameterisation, however, may need more work. While diffusion parameters and partition coefficients for pristine polymers are available, chemical exchange kinetics for microplastics under conditions of weathering, degradation and biofilm formation in the marine environment are poorly understood. Chemical exchange in the GIT has been investigated using artificial gut fluids, but dedicated dietary exposure experiments may provide better parameterisations for a wider range of chemicals. Hazard assessment of plastic-associated chemicals should ideally not only focus on particular biota and chemicals, but also use a systems approach accounting for all exposure pathways, including food web magnification and chemical mixtures. It is most plausible that marine organisms experience a trade-off between negative effects of chemical transfer from additives to the organism, and positive effects of attenuation of POP bioaccumulation, upon ingestion of microplastic (Koelmans et al. 2014b). In this respect, experimental model-validation studies using contaminated organisms and clean plastic may be as important to advance the science as most present studies that use an inverse gradient. Finally, a better quantitative understanding is needed with respect to the role of microplastic ingestion in the chemical transfer of degradable compounds. As recently pointed out by Rochman et al. (2013b), degradable compounds such as PAH and PBDEs are known to biomagnify less from prey due to degradation in the water column or metabolization by the organism or by prey species lower in the marine food web (e.g. Di Paolo et al. 2010). Because these chemicals would be preserved by sorption to microplastic, this could increase the relative role of microplastic ingestion as a relevant pathway for these chemicals. This means that the aforementioned effect of suppression of bioaccumulation of POPs would be less relevant for these degradable compounds. 
Open Access This chapter is distributed under the terms of the Creative Commons Attribution Noncommercial License, which permits any noncommercial use, distribution, and reproduction in any medium, provided the original author(s) and source are credited.

\section{References}

Andrady, A. L. (2011). Microplastics in the marine environment. Marine Pollution Bulletin, 62, $1596-1605$.

Andrady, A.L. (2015). Persistence of plastic litter in the oceans. In M. Bergmann, L. Gutow \& M. Klages (Eds.), Marine anthropogenic litter (pp. 57-72), Berlin: Springer.

Bakir, A., Rowland, S. J., \& Thompson, R. C. (2014). Enhanced desorption of persistent organic pollutants from microplastics under simulated physiological conditions. Environmental Pollution, 185, 16-23.

Besseling, E., Wegner, A., Foekema, E. M., van den Heuvel-Greve, M. J., \& Koelmans, A. A. (2013). Effects of microplastic on performance and PCB bioaccumulation by the lugworm Arenicola marina (L.). Environmental Science and Technology, 47, 593-600.

Browne, M. A., Niven, S. J., Galloway, T. S., Rowland, S. J., \& Thompson, R. C. (2013). Microplastic moves pollutants and additives to worms, reducing functions linked to health and biodiversity. Current Biology, 23, 2388-2392.

Chua, E. M., Shimeta, J., Nugegoda, D., Morrison, P. D., \& Clarke, B. O. (2014). Assimilation of Polybrominated diphenyl ethers from microplastics by the marine amphipod, Allorchestes compressa. Environmental Science and Technology, 48, 8127-8134.

Derraik, J. G. B. (2002). The pollution of the marine environment by plastic debris: a review. Marine Pollution Bulletin, 44(9), 842-852.

Di Paolo, C., Gandhi, N., Bhavsar, S., Van den Heuvel-Greve, M., \& Koelmans, A. A. (2010). Black carbon-inclusive multichemical modeling of PBDE and PCB biomagnification in estuarine food webs. Environmental Science and Technology, 44, 7548-7554.

Di Toro, D. M., Mahony, J. D., \& Gonzalez, A. M. (1996). Particle oxidation model of synthetic FeS and sediment acid-volatile sulfide. Environmental Toxicology and Chemistry, 15, 2156-2167.

Endo, S., Yuyama, M., \& Takada, H. (2013). Desorption kinetics of hydrophobic organic contaminants from marine plastic pellets. Marine Pollution Bulletin, 74, 125-131.

Foekema, E. M., De Gruijter, C., Mergia, M. T., Murk, A. J., van Franeker, J. A., \& Koelmans, A. A. (2013). Plastic in North Sea Fish. Environmental Science and Technology, 47, 8818-8824.

Gouin, T., Roche, N., Lohmann, R., \& Hodges, G. (2011). A thermodynamic approach for assessing the environmental exposure of chemicals absorbed to microplastic. Environmental Science and Technology, 45(4), 1466-1472.

Gschwend, P. M., \& Wu, S. C. (1985). On the constancy of sediment-water partition coefficients of hydrophobic organic pollutants. Environmental Science and Technology, 19, 90-96.

Hale, S. E., Martin, T. J., Goss, K. U., Arp, H. P. H., \& Werner, D. (2010). Partitioning of organochlorine pesticides from water to polyethylene passive samplers. Environmental Pollution, 158(7), 2511-2517.

Hammer, J., Kraak, M. H., \& Parsons, J. R. (2012). Plastics in the marine environment: the dark side of a modern gift. Reviews of Environmental Contamination and Toxicology, 2012(220), 1-44.

Hardy, J. T., Coley, J. A., Antrim, L. D., \& Kiesser, S. L. (1988). A hydrophobic large-volume sampler for collecting aquatic surface microlayers: Characterization and comparison with the glass plate method. Canadian Journal of Fisheries and Aquatic Sciences, 45, 822-826.

Hardy, J. T., Crecelius, E. A., Antrim, L. D., Kiesser, S. L., \& Broadhurst, V. L. (1990). Aquatic surface microlayer contamination in Chesapeake Bay. Marine Chemistry, 28, 333-351.

Harshvardhan, K., \& Jha, B. (2013). Biodegradation of low-density polyethylene by marine bacteria from pelagic waters, Arabian Sea, India. Marine Pollution Bulletin, 77, 100-106. 
Hendriks, A. J., Van der Linde, A., Cornelissen, G., \& Sijm, D. T. H. M. (2001). The power of size. 1. Rate constants and equilibrium ratios for accumulation of organic substances related to octanol-water partition ratio and species weight. Environmental Toxicology and Chemistry, 20, 1399-1420.

Hirai, H., Takada, H., Ogata, Y., Yamashita, R., Mizukawa, K., Saha, M., et al. (2011). Organic micropollutants in marine plastics debris from the open ocean and remote and urban beaches. Marine Pollution Bulletin, 62(8), 1683-1692.

Janssen, E. M. L., Croteau, M. N., Luoma, S. N., \& Luthy, R. G. (2010). Measurement and modeling of polychlorinated biphenyl bioaccumulation from sediment for the marine polychaete Neanthes arenaceodentata and response to sorbent amendment. Environmental Science \& Technology, 44, 2857-2863.

Koelmans, A. A., Besseling, E., \& Shim, W. J. (2015). Nanoplastics in the aquatic environment. In M. Bergmann, L. Gutow \& M. Klages (Eds.) Marine anthropogenic litter (pp. 329-344). Berlin: Springer.

Koelmans, A. A., Besseling, E., Wegner, A., \& Foekema, E. M. (2013a). Plastic as a carrier of POPs to aquatic organisms: A model analysis. Environmental Science \& Technology, 47, 7812-7820.

Koelmans, A. A., Besseling, E., Wegner, A., \& Foekema, E. M. (2013b). Correction to plastic as a carrier of POPs to aquatic organisms: A model analysis. Environmental Science \& Technology, 47, 8992-8993.

Koelmans, A. A., Gouin, T., Thompson, R., Wallace, N., \& Arthur, C. (2014a). Plastics in the marine environment. Environmental Toxicology and Chemistry, 33, 5-10.

Koelmans, A. A., Besseling, E., \& Foekema, E. M. (2014b). Leaching of plastic additives to marine organisms. Environmental Pollution, 187, 49-54.

Kühn, S., Bravo Rebolledo, E. L., \& van Franeker, J. A. (2015). Deleterious effects of litter on marine life. In M. Bergmann, L. Gutow \& M. Klages (Eds.), Marine anthropogenic litter (pp. 75-116), Berlin: Springer.

Lusher, A. (2015). Microplastics in the marine environment: Distribution, interactions and effects. In M. Bergmann, L. Gutow \& M. Klages (Eds.), Marine anthropogenic litter (pp. 245-312), Berlin: Springer.

Ogata, Y., Takada, H., Mizukawa, K., Hirai, H., Iwasa, S., Endo, S., et al. (2009). International pellet watch: Global monitoring of persistent organic pollutants (POPs) in coastal waters. 1. Initial phase data on PCBs, DDTs, and HCHs. Marine Pollution Bulletin, 58(10), 1437-1446.

Rochman, C. M. (2015). The complex mixture, fate and toxicity of chemicals associated with plastic debris in the marine environment. In M. Bergmann, L. Gutow \& M. Klages (Eds.), Marine anthropogenic litter (pp. 117-140), Berlin: Springer.

Rochman, C. M., Hoh, E., Hentschel, B. T., \& Kaye, S. (2013a). Long-term field measurement of sorption of organic contaminants to five types of plastic pellets: Implications for plastic marine debris. Environmental Science and Technology, 47, 1646-1654.

Rochman, C. M., Hoh, E., Kurobe, T., \& Teh, S. J. (2013b). Ingested plastic transfers hazardous chemicals to fish and induces hepatic stress. Scientific Reports, 3(3263), 1-7.

Schwarzenbach, R. P., Gschwend, P. M., \& Imboden, D. M. (2003). Environmental organic chemistry (2nd ed.). Wiley-Interscience, London.

Teuten, E. L., Rowland, S. J., Galloway, T. S., \& Thompson, R. C. (2007). Potential for plastics to transport hydrophobic contaminants. Environmental Science and Technology, 41, 7759-7764.

Teuten, E. L., Saquing, J. M., Knappe, D. R. U., Barlaz, M. A., Jonsson, S., Björn, A., et al. (2009). Transport and release of chemicals from plastics to the environment and to wildlife. Philosophical Transactions of the Royal Society B, 364(1526), 2027-2045.

Thomann, R. V., Connolly, J. P., \& Parkerton, T. F. (1992). An equilibrium model of organic chemical accumulation in aquatic food webs with sediment interaction. Environmental Toxicology and Chemistry, 11, 615-629.

Van Noort, P. C. M., \& Koelmans, A. A. (2012). Non-equilibrium of organic compounds in sediment-water systems. Consequences for risk assessment and remediation measures. Environmental Science and Technology, 46, 10900-10908. 
Velzeboer, I., Kwadijk, C. J. A. F., \& Koelmans, A. A. (2014). Strong sorption of PCBs to nanoplastics, microplastics, carbon nanotubes and fullerenes. Environmental Science and Technology, 48, 4869-4876.

Weisbrod, A., Woodburn, K., Koelmans, A. A., Parkerton, T., McElroy, A., \& Borgå, K. (2009). Evaluation of bioaccumulation using in-vivo laboratory and field studies. Integrated Environmental Assessment and Management, 5, 598-623.

Wurl, O., Karuppiah, S., \& Obbard, J. P. (2006). The role of the sea-surface microlayer in the air-sea gas exchange or organochlorine compounds. Science of the Total Environment, 369, 333-343. 